\title{
Pour un nouveau paradigme traductionnel : les traductions exogènes et endogènes
}

Benoit Doyon-Gosselin

Université Laval

D’entrée de jeu, précisons que mon apport à la réflexion globale que sous-tend la problématique de la traduction en milieu minoritaire au Canada permettra de mieux saisir les enjeux novateurs sur un aspect particulier de la traduction. En effet, si l'on s'est beaucoup intéressé à des traductions problématiques, si l'on s'est concentré à évaluer différentes traductions d'un même ouvrage ou encore si l'on se penche depuis peu sur les traductions de romans utilisant une langue hybride (par exemple, le chiac chez France Daigle), on s'est beaucoup moins soucié de la relation entre l'auteur et le traducteur. Cet objet d'étude relativement récent coïncide avec les discours sur les relations asymétriques: colonisateur/colonisé, homme/femme, centre/périphérie, etc. À ce sujet, le livre Le métier du double: portraits de traductrices et traducteurs littéraires publié en 2005 sous la direction d'Agnès Whitfield s'avère des plus pertinents. Pour de multiples raisons que je souhaite maintenant exposer, il s'avère que l'étude de cette relation revêt souvent, mais pas toujours, une importance capitale en milieu minoritaire. Ainsi, bien que chaque traduction, technique ou littéraire, soit unique, j'estime que l'on pourrait déterminer deux grands types de traduction : les traductions exogènes et endogènes. Je souhaite donc, à l'aide d'exemples, expliciter ces types de traduction pour laisser à d'autres le soin d'établir les possibilités opératoires de ma proposition. Après avoir défini la typologie préconisée dans cette étude, je vais me pencher sur deux œuvres en traduction de l'auteur franco-manitobain J. R. Léveillé dont l'une est exogène et l'autre endogène. Ensuite, il sera question d'un cas plus connu, celui de l'écrivaine Gabrielle Roy, et d'un cas moins connu, mais plus significatif, celui de la traductrice Jo-Anne Elder.

De prime abord, l'idée d'une traduction exogène ou endogène ne va pas de soi. Ces deux adjectifs antonymes se rattachent plutôt au domaine de la médecine, de la biologie, de la géologie ou encore de la sociologie ${ }^{1}$. Les propriétés exogènes d'un élément signifient que ce dernier provient de l'extérieur d'une communauté, d'une société ou d'un territoire. À l'opposé, les

\footnotetext{
${ }^{1}$ En sociologie, Henri Boyer qui discute des endo-identités (appartenances ethniques, régionales, etc.) et des exo-identités (appartenances communautaires étrangères) dans De l'autre côté $d u$ discours (2003: 61).
} 
propriétés endogènes d'un élément signifient que ce dernier prend naissance à l'intérieur d'une communauté, d'un organisme ou d'un territoire sous l'impulsion de causes internes. Comment alors peut-on parler de traduction exogène ou endogène? Avant de répondre à cette question centrale, il faut tout d'abord définir le réseau de base, le cadre de référence de toute traduction.

Par réseau de traduction, je définis la relation entre le traducteur et l'auteur original qui repose évidemment sur le texte à traduire. Cette relation peut être très limitée ou encore, elle peut très bien être inexistante, mais imaginaire ou imaginée dans le cas où l'on traduit des textes anciens ou lorsque l'auteur est décédé. Ainsi, au-delà des visées commerciales de la traduction et du rôle des maisons d'édition qui contrôlent la traduction des œuvres appartenant à leur catalogue, cette relation entre le traducteur et l'auteur, liée par le texte, est à la base de toute traduction, littéraire ou non. L'auteur doit cependant être considéré dans une acception plus large. Par exemple, dans les cas de traductions techniques, un manuel d'utilisation quelconque, le traducteur connaîtra habituellement un point de contact faisant partie de la compagnie pour répondre à ses questions. Ce point de contact pourrait être l'auteur du texte, mais pas nécessairement. Lorsqu'il s'agit d'une traduction littéraire, l'auteur de l'œuvre demeure la référence lorsque l'on souhaite régler des problèmes de compréhension du texte à traduire. Ayant établi une définition sommaire de la dynamique auteur-traducteur-traduction, on peut maintenant expliciter la typologie centrale de ma réflexion.

Une traduction exogène est, au départ, extérieure au réseau de traduction. Il s'agit du type le plus courant de traduction. Dans le monde littéraire, une maison d'édition décide qu'une œuvre importante, qui a connu un succès commercial, qui a gagné un prix littéraire prestigieux (critère fondamental pour la traduction à l'étranger) ou qui possède un intérêt plutôt universel, décide de faire traduire l'œuvre en question. Ou alors, une maison d'édition étrangère, pour les mêmes raisons, décide d'acheter les droits de traduction d'une œuvre. Il va sans dire que les traductions techniques sont toujours exogènes. Les compagnies traduisent des manuels parce que la loi l'exige dans certains pays ou encore parce que la traduction fait partie des stratégies de marketing. Le Gouvernement du Canada le fait pour respecter les droits des deux peuples fondateurs. Pour comprendre où je veux en venir, considérons un cas littéraire semblable à des milliers d'autres. Le choix de traduire un roman d'Albert Camus, par exemple, est extérieur au réseau. Nonobstant les droits de traduction qui doivent être signés par l'auteur ou son agent, ce sont véritablement les 
maisons d'édition de langue étrangère qui organisent tout le travail de traduction avec l'appui de critiques littéraires, de professeurs d'universités et de traducteurs littéraires professionnels. Bref, l'intérêt de traduire vient souvent de l'extérieur, même si, évidemment, la maison d'édition française d'Albert Camus tente de créer un tel intérêt. Ce même phénomène se produit si on prend les romans à succès de J. K. Rowling ou encore, plus près de chez nous, Un dimanche à la piscine à Kigali de Gil Courtemanche et La petite fille qui aimait trop les allumettes de Gaétan Soucy. En ce qui concerne Camus, Rowling, Courtemanche et Soucy, on pourrait alors parler de traduction exogène. L'intérêt de traduire et l'acte de traduction ne proviennent pas du réseau de traduction.

En plus de provenir de l'extérieur du réseau, les traductions exogènes possèdent d'autres propriétés qu'il faut maintenant déterminer.

1) Elles tendent vers le capital commercial. On effectue ce type de traduction en raison des retombées économiques possibles.

2) La relation professionnelle qui unit le traducteur et l'auteur s'avère plus souvent qu'autrement minimale même si le traducteur peut exiger des précisions à l'auteur.

3) Liée à la deuxième propriété, la révision finale de la traduction, effectuée par l'auteur original, tend à être minimale ou alors inexistante. Cela ne veut pas dire qu'un auteur ne lira pas la traduction, mais force est d'admettre qu'un auteur québécois ou français ne pourra généralement pas relire une traduction dans une langue qu'il ne maîtrise pas suffisamment.

4) Dans le monde littéraire, les traductions exogènes sont normalement liées aux œuvres classiques et aux écrivains canonisés des littératures nationales.

Ces quatre propriétés, loin d'être exhaustives, rendent bien compte du caractère exogène d'une traduction. Qu'en est-il alors de la traduction endogène, ce type de traduction peu étudié mais tout aussi intéressant pour le chercheur en milieu minoritaire?

Une traduction endogène provient de l'intérieur du réseau. C'est-à-dire que l'auteur ou alors le traducteur est à l'origine de la traduction. Ce type de traduction se produit plus souvent dans les milieux où les cultures sont en contact. En raison du rapport particulier que l'auteur minoritaire entretient avec la langue ou la société majoritaire, il croit que son roman ou sa poésie mérite d'être traduit. Dans un autre cas, un traducteur se trouve des affinités particulières avec une œuvre qu'il souhaite faire découvrir aux lecteurs 
appartenant à la société majoritaire. Même si les exemples de ce type de traduction abondent en Acadie, en Ontario et ailleurs, il va sans dire que les traductions endogènes ne sont pas légion lorsque comparées avec les traductions exogènes. Malgré ce constat ou peut-être en raison de celui-ci, les traductions endogènes, moins dans leurs résultats que dans le processus traductionnel, s'avèrent intéressantes en raison $\mathrm{du}$ contexte contemporain marqué par l'interculturalité et la mondialisation. Intéressantes, elles le sont d'abord et avant tout parce qu'elles possèdent des propriétés particulières :

1) Elles tendent à être plus symboliques que commerciales. On effectue ce type de traduction en raison des affinités particulières que l'on possède avec l'œuvre ou parce que l'on souhaite faire découvrir une œuvre à la population majoritaire.

2) La relation qui unit le traducteur et l'auteur s'avère plus souvent qu'autrement très importante. Les deux parties travaillent en collaboration. Il peut même y avoir une relation d'amitié préexistante à la traduction ou alors cette amitié finit par prendre de l'ampleur au fil des échanges.

3) Liée à la deuxième propriété, la révision finale de la traduction, effectuée par l'auteur original, tend à être importante. Il ne faut cependant pas généraliser. Certains auteurs s'intéressent peu à réviser la traduction de leur œuvre. Tout de même, dans le cas d'une traduction endogène, où l'auteur original possède habituellement la langue de l'autre, la révision par l'auteur est monnaie courante. Cela ne veut pas dire qu'un auteur minera le travail de traduction, mais la relation particulière entre le traducteur et l'auteur signifie habituellement une étroite collaboration.

4) Dans le monde littéraire, les traductions endogènes sont normalement liées à des auteurs qui entretiennent un rapport privilégié avec la langue et la culture d'arrivée.

Pour mieux expliquer l'idée de traduction exogène et de traduction endogène, je souhaite maintenant traiter de l'œuvre traduite du franco-manitobain J. R. Léveillé dans une perspective sociologique inspirée de Henri Boyer (2003), c'està-dire en décrivant les étapes qui ont mené à la traduction de son roman Le Soleil du lac qui se couche ainsi que celles qui ont permis à la novella New York trip de paraître en anglais. Je ne m'intéresserai donc pas au résultat de la traduction, mais au processus.

\section{Un cas exemplaire : J. R. Léveillé}


En 2001, une première a lieu au Manitoba. Au Centre culturel franco-manitobain, on lance le nouveau roman du franco-manitobain J. R. Léveillé, Le soleil du lac qui se couche, en français et en anglais. Il ne s'agit pas d'une édition bilingue comme on en publie parfois dans l'Ouest canadien. On parle ici de deux livres distincts dont la version anglaise The Setting Lake Sun traduite par S. E. Stewart parait sous les auspices de Signature Editions. Les deux livres comportent plusieurs différences autant sur le plan matériel que dans les choix formels, mais il n'en demeure pas moins que pour la première fois un livre d'un auteur francomanitobain est lancé en même temps dans sa version originale française et dans sa version anglaise. Comment alors Le soleil du lac qui se couche est-il devenu The Setting Lake Sun? Malgré l'importance de son œuvre, qui souhaite véritablement traduire J. R. Léveillé ? Qui, au Canada anglais, s'intéresse à J. R. Léveillé au point de vouloir le traduire?

Les Éditions du Blé, principal éditeur des œuvres de Léveillé, souhaitaient depuis longtemps qu'un livre de leur auteur le plus reconnu soit traduit. Lucien Chaput, le directeur de la maison d'édition, pilotait le projet avec l'accord de Léveillé. Le cinquième roman de Léveillé s'avérait le choix le plus logique, car la trame narrative se situait au Manitoba. Il s'agissait en fait du premier roman «manitobain» de l'auteur. Le soleil du lac qui se couche raconte l'histoire toute simple d'une Métisse âgée de vingt ans qui rencontre un vieux poète japonais âgé de soixante-quatre ans. D'abord fascinée, Angèle tombe peu à peu sous le charme d'Ueno Takami qu'elle rencontre dans la ville de Winnipeg. Ensemble, ils voyageront en camion $4 \times 4$ vers Setting Lake où se situe la demeure du vieil homme. À travers l'enseignement tout en douceur de vieux poète, Angèle deviendra un être à part entière n'ayant plus honte de son héritage métis. De par ce thème universel, on comprend alors l'intérêt que pourrait susciter ce roman pour le lectorat anglophone au Manitoba, mais également hors des frontières de la province.

Ainsi, Lucien Chaput a conclu une entente avec Signature Editions, une petite maison d'édition littéraire établie à Montréal en 1986 qui avait entre temps déménagé à Winnipeg en 1997. La traduction a été complétée rapidement par S. E. Stewart et a obtenu une mention honorable pour le Prix de traduction John Glassco. Bien que Léveillé ait eu un droit de regard sur la traduction et qu'il ait proposé quelques modifications, les délais serrés pour la publication ont fait en sorte que le résultat final ne fut que simplement satisfaisant pour l'auteur. En fait, le plus grand reproche de Léveillé quant à la traduction réside dans le choix de la traductrice d'avoir essayé de recréer un récit plus linéaire en interchangeant 
de nombreux segments (Léveillé/Doyon-Gosselin 2007). Quoiqu'il en soit, The Setting Lake Sun demeure un parfait exemple d'une traduction exogène. Certes, il ne s'agit pas d'une œuvre classique, mais d'un auteur important pour le Manitoba. Les maisons d'édition ont travaillé de concert mais l'auteur n'a pas entretenu de véritables liens avec la traductrice.

De son côté, la traduction de la novella New York trip a connu un destin passablement différent. Mentionnons tout d'abord qu'un long extrait de l'œuvre avait déjà paru dans le numéro quatre de la revue Virages. Quelques années plus tard, comme Léveillé souhaitait publier la version intégrale de New York trip, il approche Stefan Psenak des Éditions L'interligne. La maison d'édition ontarienne accepte de la publier, et le livre paraît en format bilingue en 2003. Cependant, L'Interligne ne s'est aucunement occupée de la traduction. En fait, lorsque Léveillé a proposé de publier une version anglaise dans le même volume, la maison d'édition a expliqué qu'elle était prête à assumer les coûts de la version originale, mais que Léveillé devait s'occuper de financer la version anglaise. Ainsi, à l'intérieur du livre, on apprend que les droits d'auteur de la traduction appartiennent à la maison d'édition Ink Inc., un petit projet d'édition dans les deux langues que J. R. Léveillé et son frère Bernard ont entrepris en 1987 afin de réaliser, à l'occasion, des publications qui peut-être ne verraient pas autrement le jour ou pour assurer des publications ponctuelles, ce qu'une maison établie peut rarement se permettre en raison de son programme d'édition annuel. Ainsi, c'est l'auteur lui-même, à travers sa maison d'édition, qui a assumé les frais de la publication de la traduction (Léveillé/Doyon-Gosselin 2007).

En ce qui concerne le choix du traducteur, Léveillé s'est tourné vers un bon ami, Guy Gauthier, un Franco-manitobain établi à New York depuis plusieurs décennies. De 1969 à 1975, Gauthier a écrit une vingtaine de pièces qui ont été montées Off-Off-Broadway. Il est donc autant à l'aise en français qu'en anglais. Comme il existe déjà une amitié profonde entre les deux auteurs, le travail de traduction en devient un de collaboration. Léveillé et Gauthier discuteront souvent de l'œuvre pour permettre aux deux parties d'en arriver à la meilleure traduction possible. Cette relation ressemble par ailleurs à celles évoquées dans le cas de la traduction féministe au Québec. Bref, il semble clair que dans le cas de New York trip/Trip, nous avons affaire à une traduction endogène, l'auteur étant à l'origine de l'intention de traduire l'œuvre première. Il faut comprendre que parce que l'auteur est bien connu dans la communauté anglophone manitobaine après quarante ans de carrière, il décide lui-même que son œuvre pourrait rayonner en traduction anglaise. En ce sens, Léveillé 
demeure peut-être un cas unique au Manitoba. En effet, c'est surtout à cause de son rapport avec la communauté anglophone du Manitoba que deux de ses trois dernières œuvres ont paru en traduction.

\section{Deux autres cas exemplaires}

Un cas bien documenté est celui des traductions de l'œuvre de Gabrielle Roy. Comme le relate François Ricard dans sa superbe biographie Gabrielle Roy. Une vie qui a paru en 2000, les premières œuvres de Roy, à part Bonheur d'occasion, ont été traduites par Harry Lorin Binsse. Bien qu'extrêmement compétent, le traducteur travaille très lentement. De plus, il a été imposé par la maison d'édition de langue anglaise. Lorsqu'un changement survient au sujet des maisons d'édition publiant l'œuvre de Roy en anglais, Binsse est congédié. C'est alors que Roy suggère elle-même Joyce Marshall comme traductrice de ses œuvres. Depuis la fin des années 1950, les deux femmes sont de bonnes amies qui partagent les mêmes sensibilités littéraires. Ainsi, lorsque vient le temps de traduire La route d'Altamont, Marshall a accès au manuscrit avant même que la version française ne soit publiée. De plus, Ricard précise que Roy «collabore ensuite au travail de traduction [...]» (2000: 419). S'ensuit alors une longue collaboration entre l'auteur et sa traductrice qui se termine en 1973 lorsque Marshall souhaite privilégier sa propre carrière littéraire.

Dans l'esprit de Gabrielle Roy, cette collaboration s'avère des plus fructueuses, car en tant que première grande écrivaine canadienne (au sens où elle est lue et connue également par la population de la langue anglaise et de langue française), elle accorde une importance de premier plan à la diffusion de son œuvre en anglais. Elle peut donc se permettre d'intervenir à toutes les étapes de la traduction, sans véritablement froisser son amie de longue date. Il s'agit ici d'une traduction endogène, initiée par l'auteur, liée au fait que Roy entretient une excellente relation avec la langue et la culture d'arrivée. Il devient donc intéressant d'étudier la correspondance entre l'auteur et la traductrice et, si possible, les divers manuscrits des versions traduites pour établir ce qui éloigne et rapproche les deux écrivaines. Comme le fait remarquer Ricard: «[...] leur abondante correspondance constitue un très bel exemple de cette écriture à quatre mains que peut devenir, lorsque les conditions s'y prêtent, le travail de la traduction » (2000 : 494). L'étude de cette relation traductionnelle basée au départ sur amitié a été en partie réalisée par Jane Everett (2005). Cependant, il faut souligner que le choix de traduire Gabrielle Roy est loin de seulement tendre vers le symbolique. L'œuvre de Roy en anglais demeure une entreprise littéraire 
tout autant que commerciale, même si les ventes n'ont pas toujours été au rendez-vous.

Un autre cas plus récent mais moins connu de traduction endogène serait peut-être plus pertinent à relever. Jo-Anne Elder, qui réside à Fredericton au Nouveau-Brunswick, est directrice de la revue littéraire ellipse en plus d'être praticienne de la traduction. Elle a entre autres traduit le roman Moncton Mantra de Gérald Leblanc, la poésie de Robert Dickson ainsi que la poésie d'Herménégilde Chiasson. Dans le cas d'Elder, une particularité nouvelle mérite d'être soulevée. D'une part, l'initiative de la traduction revient parfois à la traductrice. C'est Elder qui a d'abord approché les auteurs pour leur demander si elle pouvait s'attaquer à la traduction d'un roman ou d'un recueil en particulier. On se rend compte de tout le travail symbolique que revêt cette entreprise littéraire. La traduction endogène, qui tend justement vers le symbolique au détriment du commercial, trouve habituellement son origine dans la personne de l'auteur, mais il arrive parfois, que le traducteur offre ses services à l'auteur en raison par exemple des affinités de leur écriture respective. Au demeurant, Elder elle-même, dans ses articles ${ }^{2}$, traite fréquemment de sa relation avec les auteurs lorsqu'elle agit en tant que traductrice. Selon Elder, la traduction endogène signifie d'abord et avant tout que le traducteur doit aimer profondément le texte traduit au point de l'intégrer dans sa vie. En outre, elle indique qu'il doit y avoir une collaboration entre tous les acteurs littéraires (maison d'édition, auteur, traducteur). Enfin, un respect mutuel doit exister entre l'auteur et le traducteur (Elder/Doyon-Gosselin 2008). Dans ce cas précis, malgré les quelques réserves liées au manque de distanciation entre le sujet et son objet, la traductrice et l'auteur seraient peut-être les mieux placés pour parler de la relation qui les unit. On en apprendrait alors sur les bénéfices mutuels qui découlent de celle-ci. Cet essai l'invite ainsi à relever le défi.

\section{Conclusion}

Afin de conclure, rappelons brièvement, à l'aide d'un tableau, les caractéristiques des traductions de type exogène et de type endogène.

\begin{tabular}{|l|l|}
\hline Traduction exogène & Traduction endogène \\
\hline Extérieur du réseau de traduction & Intérieur du réseau de traduction \\
\hline
\end{tabular}

\footnotetext{
${ }^{2}$ Voir entre autres Jo-Anne Elder (2007), « Des rêves inachevés aux projets en perpétuel devenir : traduire l'Acadie ici et ailleurs » et (2006) « Côte à côte : traduction et diffusion de la littérature acadienne ».
} 


\begin{tabular}{|l|l|}
\hline Entreprise plutôt commerciale & Entreprise plutôt symbolique \\
\hline $\begin{array}{l}\text { Collaboration entre le traducteur et } \\
\text { l'auteur tend à être minimale }\end{array}$ & $\begin{array}{l}\text { Collaboration entre le traducteur et } \\
\text { l'auteur tend à être plus importante }\end{array}$ \\
\hline $\begin{array}{l}\text { Révision finale de l'œuvre traduite par } \\
\text { l'auteur original est généralement } \\
\text { minimale }\end{array}$ & $\begin{array}{l}\text { Révision finale de l'œuvre traduite par } \\
\text { l'auteur original tend à être importante }\end{array}$ \\
\hline $\begin{array}{l}\text { Euvres classiques et auteurs canonisés } \\
\text { des littératures nationales }\end{array}$ & $\begin{array}{l}\text { Auteurs ayant un rapport privilégié } \\
\text { avec la langue et la culture d'arrivée }\end{array}$ \\
\hline
\end{tabular}

Je souhaite maintenant revenir sur deux idées qui seront peut-être des lieux communs, mais qui me semblent capitales pour comprendre les rapports à la traduction dans le Canada francophone minoritaire. D'une part, avant même de parler de traduction, il faut se rendre à l'évidence que les écrivains francophones en milieu minoritaire, comme tous les peuples minoritaires, entretiennent des rapports particuliers avec la langue de la majorité. Il est clair que Léveillé ou Dickson ne voient pas la langue anglaise de la même façon que la majorité des grands auteurs des cultures hégémoniques. Dans cet ordre d'idées, il devient évident que J.R. Léveillé, par exemple, ne conçoit pas la traduction de ses œuvres comme une activité commerciale. On ne parle pas de rentabilité, mais bien de partage de culture, d'offrir à l'autre dans sa langue un peu de soi-même ${ }^{3}$.

D'autre part, les notions de traductions exogène et endogène s'avèrent particulièrement intéressantes dans le contexte minoritaire. À l'instar de Léveillé, le traducteur, bien qu'il soit rémunéré pour son travail, choisit de traduire une œuvre issue des littératures de l'exiguiité pour connaître et pour faire connaître l'autre. Il choisit également de traduire parce que l'œuvre lui parle, parce qu'il y gagne culturellement au change. Le même constat peut s'appliquer pour le travail de traduction de Jo-Anne Elder. Tout en se reconnaissant à travers l'autre, elle souhaite sans aucun doute faire découvrir l'autre à la culture dominante parce qu'elle aime profondément les textes choisis, comme l'a fait Susanne de Lotbinière-Harwood (Merkle 2005). En ce qui concerne plus précisément les traductions endogènes, il me semble que la relation entre l'auteur et son traducteur mériterait d'être étudiée ${ }^{4}$. Ainsi, il serait pertinent de décrire et

\footnotetext{
${ }^{3}$ À ce sujet, le parcours de Susanne de Lotbinière-Harwood est exemplaire. Voir Denise Merkle (2005), " Susanne de Lotbinière-Harwood, médiatrice culturelle "in nomad's land" ».

${ }^{4}$ L'exemple de la traduction féministe au Québec s'intéresse depuis longtemps déjà à la relation entre l'auteur et le traducteur. Voir entre autres, Aurelia Klimkiewicz (2004), « La traduction féministe : une mise en pratique du principe dialogique ». Un exemple de traduction endogène au féminin se trouve chez Nathalie Ramière (2003), «Stratégies de traduction et non-traduction dans The Widows de Suzette Mayr ». Dans un contexte plus large, voir également Agnès Whitfield
} 
d'analyser les rapports entre Jo-Anne Elder et le regretté Gérald Leblanc, entre Lola Lemire Tostevin et le regretté Robert Dickson ${ }^{5}$ ou entre Robert Mazjels et France Daigle. Il existe à mon sens une filiation qui mériterait d'être explorée.

\section{BIBLIOGRAPHIE}

BOYER, Henri (2003), De l'autre côté du discours : recherches sur le fonctionnement des représentations communautaires, Paris, L'Harmattan.

ELDER, Jo-Anne (2007), " Des rêves inachevés aux projets en perpétuel devenir : traduire l'Acadie ici et ailleurs », dans Janine Gallant, Hélène Destrempes et Jean Morency (dirs.), L'cuvre littéraire et ses inachèvements, Longueuil, Groupéditions, 259-268.

ELDER, Jo-Anne (2006), "Côte à côte : traduction et diffusion de la littérature acadienne », dans Marie-Linda Lord (dir.), L'émergence et la reconnaissance des études acadiennes: à la rencontre de Soi et de l'Autre, Moncton, Association internationale des études acadiennes, 159-178.

ELDER, Jo-Anne (1 mars 2008), correspondance personnelle, courriel envoyé à Benoit Doyon-Gosselin.

EVERETT, Jane, dir. (2005), In translation: the Gabrielle Roy-Joyce Marshall correspondence, Toronto, University of Toronto Press.

LÉVEILLÉ, J. R. (2001), Le soleil du lac qui se couche, Saint-Boniface, Éditions du Blé.

LÉVEILLÉ, J. R. (2001), The Setting Lake Sun, traduit par S. E. Stewart, Winnipeg, Signature Editions.

LÉVEILLÉ, J. R. (2003), New York trip ou Tableaux d'une exposition / New York Trip or Pictures from an Exhibition, Ottawa, Les Éditions L'interligne / Ink Inc.

LÉVEILLÉ, J. R. (février 2007), entretien téléphonique personnel avec Benoit Doyon-Gosselin.

(2005), Le métier du double : portraits de traductrices et traducteurs littéraires et (2006), Writing Between the Lines: Portraits of Canadian Anglophone Translators.

${ }^{5}$ Voir à ce sujet Judith Woodsworth (2008), « Translating Identity in Northern Ontario ». 
KLIMKIEWICZ, Aurelia (2004), «La traduction féministe : une mise en pratique du principe dialogique ", dans Aurelia Klimkiewicz et Sylvie Mongeau (dirs.), Esquisse du féminin: les conteurs d'une dérive, Montréal, CÉLAT, UQAM, Coll. « Les cahiers du CÉLAT», 87-98.

MERKLE, Denise (2005), « Susanne de Lotbinière-Harwood, médiatrice culturelle "in nomad's land" » dans Jean Morency, Hélène Destrempes, Denise Merkle et Martin Pâquet (dirs.), Des cultures en contact. Vision de l'Amérique du Nord francophone, Québec, Éditions Nota bene, Coll. « Terre américaine », 495-509.

RAMIÈRE, Nathalie (2003), «Stratégies de traduction et non-traduction dans The Widows de Suzette Mayr », TTR 16/2, 175-196.

RICARD, François (2000), Gabrielle Roy. Une vie, Montréal, Éditions du Boréal (Coll. « Boréal express » $\left.\mathrm{n}^{\circ} 110\right)$.

WHITFIELD, Agnès, dir. (2005), Le métier du double: portraits de traductrices et traducteurs littéraires, Saint-Laurent, Québec, Fides, Coll. «Nouvelles études québécoises ».

WHITFIELD, Agnès, dir. (2006), Writing Between the Lines: Portraits of Canadian Anglophone Translators, Waterloo, Ontario, Wilfrid Laurier University Press.

WOODWORTH, Judith (2008), "Translating identity in Northern Ontario », dans Denise Merkle, Jane Koustas, Glen Nichols et Sherry Simon (dirs.), Traduire depuis les marges/Translating from the Margins, Québec, Éditions Nota bene, Coll. «Terre américaine », 95-120. 